\title{
Die Bestimmung des Gesamtphosphors in Abwässern mit Ammoniummolybdat und r-Amino-2-naphthol-4-sulfonsäure («ANS-Heizmethode»)
}

(Beiträge zur chemischen Wasser- und Abwasseranalyse, Nr. 4)

Von H. R. HEGI ${ }^{1}$ )

Eidg. Anstalt für Wasserversorgung, Abwasserreinigung und Gewässerschutz an der Eidg. Technischen Hochschule, Zürich. Direktion: Prof. Dr. O. Jaag

Manuskript eingegangen am 30. Mai 1967

\section{Einleitung}

Bei der photometrischen Bestimmung von Phosphat mit Ammoniummolybdat in schwefelsaurer Lösung und Reduktion des Phosphormolybdänkomplexes zu Phosphormolybdänblau werden als Reduktionsmittel entweder Zinn-II-chlorid oder auch organische Reduktionsmittel angewandt. Die Reaktion mit Zinn-II-chlorid kommt auch in der Wasser- und Abwasseranalyse sehr häufig zur Anwendung und eignet sich wegen ihrer hohen Empfindlichkeit vor allem für sehr geringe Phosphatkonzentrationen. AmbÜHL und Schmid [1] sowie Schmid und AmbüHL [5] beispielsweise haben sich für die Anwendung von Zinn-II-chlorid entschieden, da sie im Wasser von Binnenseen meist sehr geringe Phosphatkonzentrationen mit grosser Genauigkeit bestimmen mussten. Wir haben indessen die Erfahrung gemacht, dass bei der Bestimmung des Gesamtphosphors in höheren Konzentrationen (Abwässer, Kläranlagenabflüsse, Vorfluter) die Anwendung von 1-Amino-2-naphthol-4-sulfonsäure als Reduktionsmittel zur Erreichung derselben Genauigkeit in bedeutend geringerem Masse die Einhaltung minuziöser Arbeitsbedingungen erfordert als die Anwendung von Zinn-IIchlorid. Die Vorzüge der von uns vorgeschlagenen Methode sind eine gute Reproduzierbarkeit der Messwerte, die relativ geringe Abhängigkeit der Farbintensität von der Säurekonzentration, die grosse Beständigkeit der Farbintensität während mehrerer Stunden und die geringe Störanfälligkeit gegen Eisen (wichtig bei der Kontrolle der Phosphatelimination mit Eisen-III-chlorid). Frske und SubBarow [3] haben seinerzeit die Anwendung der 1-Amino-2-naphthol-4-sulfonsäure als Reduktionsmittel bei Phosphatbestimmungen eingeführt, BARTLETT [2] publizierte eine Modifikation der Anwendung dieses Reagenz, bei welcher durch Erhitzen nach der Zugabe der Reagenzien die Farbintensität erheblich gesteigert werden kann, wobei sich bei $830 \mathrm{~nm}$ ein

1) Frau Martha Vogel-Huber sei für die gewissenhafte Ausführung eines grossen Teils der experimentellen Untersuchungen bestens gedankt. 
Maximum der Farbintensität bildet, dessen optische Dichte 7,2mal grösser sein soll als diejenige, die üblicherweise ohne Erhitzen bei $660 \mathrm{~nm}$ gemessen wird. Die nachstehende, auf den Angaben von Bartlett [2] beruhende und von uns auf die Anwendung in der Abwasseranalyse angepasste Arbeitsvorschrift hat sich während zweier Jahre in unserem Laboratorium bewährt. Die 1-Amino-2-naphthol-4-sulfonsäure wird abgekürzt als ANS bezeichnet, und wir möchten daher unsere Vorschrift als "ANS-Heizmethode» bezeichnen. Wir haben ihre Anwendung allerdings vorerst auf die Bestimmung des Gesamtphosphors beschränkt, da bei der Bestimmung des Phosphats (Orthophosphats) durch das der Reagenzienzugabe nachfolgende Erhitzen oftmals gleichzeitig vorhandene kondensierte Phosphate oder organische Phosphorverbindungen hydrolysiert und fälschlicherweise miterfasst werden könnten. Auf jeden Fall müsste eine solche Fehlermöglichkeit in Erwägung gezogen und überprüft werden, wenn man diese Vorschrift auf die Bestimmung des Orthophosphats übertragen möchte. Für die Bestimmung des Orthophosphats in Abwässern, Abflüssen usw. verwenden wir in unserem Laboratorium nach wie vor Zinn-IIchlorid als Reduktionsmittel, wobei wir nötigenfalls die Extraktion des Phosphormolybdänkomplexes mit nachfolgender Reduktion durch Zinn-II-chlorid vornehmen. Für dieses Extraktionsverfahren, durch dessen Anwendung Störungen weitgehend ausgeschaltet werden können, gehen der AASGP Committee Report [6], KLoTTER und Neussel [4] sowie die Standard Methods [7] annähernd dieselbe Arbeitsvorschrift.

Die nasse Mineralisierung (im Gegensatz zur trockenen Veraschung), z. B. mit Schwefelsäure und Wasserstoffperoxid, macht es prinzipiell möglich, den Aufschluss für die Gesamtphosphor- und die Kjeldahlstickstoffbestimmung gemeinsam vorzunehmen. Einige vergleichende Untersuchungen über verschiedene Mineralisierungsmethoden an einigen Testsubstanzen und an Abwasserproben weisen deutlich darauf hin, dass bei der Untersuchung von kommunalen Abwässern und Kläranlagenabflüssen das von uns vorgeschlagene Verfahren zur nassen Mineralisierung auch bei der Kjeldahlstickstoffbestimmung brauchbare Resultate liefert. Die in häuslichen bzw. kommunalen Abwässern zur Hauptsache vorliegenden Gruppen von organischen Phosphor- bzw. Stickstoffverbindungen lassen sich mit den üblichen Verfahren zur nassen Mineralisierung ohne Schwierigkeit zu den durch die nachfolgende Bestimmung erfassbaren Verbindungen Orthophosphat und Ammonium umsetzen; anders verhält es sich bei gewissen Industrieabwässern, welche organische Phosphor- (z.B. mit C-P-Bindungen, leichtflüchtige Verbindungen) oder Stickstoffverbindungen (aromatische oder heterozyklische Verbindungen mit ringständigem Stickstoff) enthalten, die durch das vorgeschlagene - aber wohl auch durch alle oder doch die meisten anderen - Mineralisierungsverfahren unter Anwendung von Schwefelsäure mit Zusätzen nicht vollständig erfasst werden.

\section{Arbeitsvorschrift}

\subsection{Reagenzien, besondere Geräte}

Schwefelsäure: konz. p.a.

Perhydrol: Wasserstoffperoxid 30prozentig, p.a. «Merck».

Ammoniummolybdatlösung: $50 \mathrm{~g}$ Ammoniummolybdat p.a. werden in entsalztem Wasser zu $1000 \mathrm{ml}$ gelöst. Das Reagenz ist im Dunkeln aufzubewahren. 
ANS-Reagenz: $15 \mathrm{~g}$ Natriummetabisulfit (= Natriumdisulfit $=\mathrm{Na}_{2} \mathrm{~S}_{2} \mathrm{O}_{5}$ ) werden in entsalztem Wasser zu $100 \mathrm{ml}$ gelöst. $\mathrm{Zu}$ dieser Lösung werden 0,1 g 1-Amino-2naphthol-4-sulfonsäure (ANS) zugegeben (es ist eine höchstens hellrosa bis hellorange, nicht aber dunkelviolett gefärbte Substanz zu verwenden). Die Mischung ist soweit zu erwärmen, bis die ANS in Lösung geht und hierauf sofort abzukühlen. Dann wird $1 \mathrm{~g}$ Natriumsulfit wasserhaltig p.a. $\left(\mathrm{Na}_{2} \mathrm{SO}_{3} \cdot 7 \mathrm{H}_{2} \mathrm{O}\right)$ in der Mischung gelöst. Das Reagenz ist im Dunkeln und kühl aufzubewahren und ist so etwa eine Woche lang haltbar.

Schwefelsäure 1:5: Zu etwa $300 \mathrm{ml}$ entsalztem Wasser sind $100 \mathrm{ml}$ Schwefelsäure konz. p. a. zuzugeben. Nach dem Erkalten der Mischung ist mit entsalztem Wasser auf $500 \mathrm{ml} \mathrm{zu}$ ergänzen.

Phosphatstammlösung: 4,393 g Kaliumdihydrogenphosphat $\left(\mathrm{KH}_{2} \mathrm{PO}_{4}\right)$ p.a. werden in entsalztem Wasser gelöst, $1 \mathrm{ml}$ konz. Schwefelsäure p.a. zugesetzt und zu 1 Liter aufgefüllt. $1 \mathrm{ml}$ Phosphatstammlösung enthält $1 \mathrm{mg} \mathrm{P}$.

Kühlaufsätze: Reagenzgläser von etwa $15 \mathrm{~mm}$ lichter Weite, die auf mittlerer Höhe durch Ausblasen kugelförmig erweitert worden sind, so dass die entstandene Kugel einen Durchmesser von 30-35 mm aufweist. Die Kühlaufsätze werden vor Gebrauch bis knapp über die Hälfte mit entsalztem Wasser gefüllt.

Reagenzgläser mit Glasstopfen: Inhalt $20 \mathrm{ml}$. Sie können graduiert sein, doch soll das Einmessen der Reagenzlösungen und des Verdünnungswassers grundsätzlich mit Pipetten vorgenommen werden; die Graduierung diene lediglich als Kontrolle der Einmasse.

Siedesteine: beispielsweise kleine Stücke einer grobporigen Glassinterplatte.

\section{2 Ausführung der nassen Mineralisierung}

Es ist ein dem zu erwartenden Gesamtphosphorgehalt der Probe entsprechendes Einmass an Wasserprobe zu wählen. Die eingemessene Probe enthalte nach Möglichkeit 0,002-0,10 mg P; bei sehr inhomogenen phosphatreichen Proben ist mehr einzumessen. Von Wasserproben, die sehr phosphatarm sind, muss nach Zusatz der untenerwähnten $2 \mathrm{ml}$ Schwefelsäure p. a. ein grosses Volumen (möglicherweise über $100 \mathrm{ml}$ ) eingedampft werden.

Das Probeneinmass wird mit $2 \mathrm{ml}$ konz. Schwefelsäure p.a. nach Zugabe eines Siedesteines in einem Kjeldahlkolben bis zum völligen Verschwinden des Wassers eingedampft. Wenn das Wasser beinahe völlig abgedampft ist, sind Verluste durch Spritzen möglich. Dabei ist dann so wenig zu erhitzen, dass das Wasser noch abdunstet, die Flüssigkeit aber nicht mehr siedet und sich keine Schwaden von Schwefelsäuredämpfen entwickeln. Verkohlung ist möglichst zu vermeiden. Nach teilweisem Abkühlen werden 2 Tropfen Perhydrol zugesetzt und etwa 10 Minuten lang bis knapp zum Sieden erhitzt, wobei darauf zu achten ist, dass die oberste Partie des Kolbenhalses nicht heiss wird. Bei weithalsigen Kolben empfiehlt es sich, einen Kühlaufsatz zu verwenden. Kühlaufsätze verschiedener Kolben dürfen untereinander nicht ausgetauscht werden. Die Perhydrolzugabe und das nachherige Erhitzen sind zu wiederholen, bis die Flüssigkeit völlig farblos geworden ist und das darin eventuell enthaltene Ungelöste weiss ist. Dann sind nach teilweisem Abkühlen nochmals 2 Tropfen 
Perhydrol zuzugeben, und es ist noch während etwa 30 Minuten zu erhitzen. Nach fast völligem Erkaltenlassen werden etwa $50 \mathrm{ml}$ entsalztes Wasser unter Abspülen des Kühlaufsatzes zugegeben und die Mischung während 15 Minuten zu leichtem Sieden erhitzt (Umwandlung von möglicherweise gebildetem Pyrophosphat in Orthophosphat), wobei zu beachten ist, dass nicht auf weniger als etwa $20 \mathrm{ml}$ eingeengt wird (nötigenfalls entsalztes Wasser nachgeben).

\subsection{Ausführung der photometrischen Bestimmung}

Die gesamte Aufschlusslösung enthält von der Mineralisierung her $2 \mathrm{ml}$ konz. Schwefelsäure. Sie ist mit entsalztem Wasser auf genau $50 \mathrm{ml} \mathrm{zu}$ ergänzen. Ein Teil der auf $50 \mathrm{ml}$ verdünnten Lösung kann zur Bestimmung des Kjeldahlstickstoffes verwendet werden. Von dieser Verdünnung ist ein aliquoter Teil, der höchstens $10 \mathrm{ml}$ betragen sowie höchstens 0,01 mg $\mathrm{P}$ (absolut) enthalten darf, zur photometrischen Bestimmung in ein Reagenzglas mit Glasstopfen einzumessen. Der aliquote Teil der Aufschlusslösung, der zur Bestimmung verwendet wird, ist auf $12 \mathrm{ml}$ Gesamtvolumen sowie auf einen Gehalt von $0,4 \mathrm{ml}$ konz. Schwefelsäure zu ergänzen.

Beispiele:

a) Verwendung von $10 \mathrm{ml}$ Aufschlusslösung (falls $10 \mathrm{ml}$ Aufschlusslösung höchstens $0,01 \mathrm{mg} P$ enthalten): die $10 \mathrm{ml}$ Aufschlusslösung enthalten bereits $0,4 \mathrm{ml} \mathrm{konz}$. Schwefelsäure. Es sind $2 \mathrm{ml}$ entsalztes Wasser zuzugeben.

b) Verwendung von $5 \mathrm{ml}$ Aufschlusslösung (falls $10 \mathrm{ml}$ Aufschlusslösung 0,01-0,02 mg $\mathrm{P}$ enthalten): Die $5 \mathrm{ml}$ Aufschlusslösung enthalten bereits $0,2 \mathrm{ml}$ konz. Schwefelsäure und sind mit $1 \mathrm{ml}$ Schwefelsäure 1:5 (enthaltend 0,2 $\mathrm{ml} \mathrm{konz.}$ Schwefelsäure) und $6 \mathrm{ml}$ entsalztem Wasser zu versetzen.

c) Verwendung von $2 \mathrm{ml}$ Aufschlusslösung (falls $10 \mathrm{ml}$ Aufschlusslösung 0,02-0,05 mg $\mathrm{P}$ enthalten): Die $2 \mathrm{ml}$ Aufschlusslösung, enthalten bereits $0,08 \mathrm{ml} \mathrm{konz}$. Schwefelsäure und sind mit 1,6 ml Schwefelsäure 1:5 (enthaltend 0,32 $\mathrm{ml} \mathrm{konz.} \mathrm{Schwefel-}$ säure) und $8,4 \mathrm{ml}$ entsalztem Wasser zu versetzen.

Gleichzeitig mit den Proben sind eine Reagenzienblindprobe sowie P-Standards anzusetzen. Die Reagenzienblindprobe enthält $10 \mathrm{ml}$ entsalztes Wasser und $2 \mathrm{ml}$ Schwefelsäure 1:5; die Standards (Einmasse einer Verdünnung der Phosphatstammlösung) sind mit $2 \mathrm{ml}$ Schwefelsäure 1:5 zu versetzen und mit entsalztem Wasser auf $12 \mathrm{ml}$ zu ergänzen. Nach dem Ergänzen auf $12 \mathrm{ml}$ werden $0,5 \mathrm{ml}$ Ammoniummolybdatlösung zugegeben und geschüttelt, dann 0,5 ml ANS-Reagenz zugegeben und wiederum geschüttelt. Die Reagenzgläser sind in ein siedendes Wasserbad zu stellen und die Glasstopfen lose aufzusetzen. Ein weiteres Reagenzglas wird mit etwa $12 \mathrm{ml}$ Wasser versetzt und mit einem Thermometer versehen. Nachdem die Temperatur auf $90^{\circ} \mathrm{C}$ gestiegen ist, werden die Reagenzgläser noch 7 Minuten im siedenden Wasserbad belassen. Hierauf werden sie in kaltes Wasser gestellt. Sogleich nach dem Erkalten kann die Extinktion bei $820 \mathrm{~nm}$ (brauchbar ist auch ein Filter, dessen maximale Durchlässigkeit im Bereich von etwa 700-800 nm liegt, doch wird dadurch die Empfindlichkeit geringer) gemessen werden. Die Extinktion bleibt während mehrerer Stunden praktisch konstant. 


\subsection{Berechnung und Angabe der Resultate}

Die Resultate werden in $\mathrm{mg} / \mathrm{l} \mathrm{P}$ angegeben.

$$
\begin{aligned}
& \operatorname{mg} \mathrm{P}(\text { in } E 2)=\frac{e_{M}}{e_{S t}} \cdot \operatorname{mg~} \mathrm{P}(\text { in } S t) \\
& \left.\operatorname{mg} \mathrm{P}(\text { in } E 1)=\frac{50}{\mathrm{ml} E 2} \cdot \operatorname{mg~} \mathrm{P} \text { (in } E 2\right) \\
& \left.\mathrm{mg} / \mathrm{l} \mathrm{P} \quad=\frac{1000}{\mathrm{ml} E 1} \cdot \mathrm{mg} \mathrm{P} \text { (in } E 1\right)
\end{aligned}
$$

$e_{M} \quad$ Extinktion der Messlösung der Probe.

$e_{S t} \quad$ Extinktion der Messlösung des Standards $S t$.

St Standard, dessen Extinktion der Extinktion der Messlösung am nächsten liegt.

E1 Einmass an Probe zur Mineralisierung.

E2 Einmass an (auf $50 \mathrm{ml}$ verdünnter) Aufschlusslösung zur photometrischen Bestimmung.

\section{Experimentelle Hinweise zur Arbeitsvorschrift}

Bei der Ausführung hielten wir uns, sofern nicht anderes vermerkt ist, an die oben beschriebene Arbeitsvorschrift.

\subsection{Spektraler Verlauf der Absorption der Messlösung}

0,7 mg/1 P. Zeiss-Spektralphotometer PMQ II, Schichtdicke $10 \mathrm{~mm}$.

\begin{tabular}{llllllll}
\hline $\mathrm{nm}$ & $e$ & $\mathrm{~nm}$ & $e$ & $\mathrm{~nm}$ & $e$ & $\mathrm{~nm}$ & $e$ \\
\hline 900 & 0,173 & 810 & 0,451 & 720 & 0,206 & 620 & 0,147 \\
850 & 0,414 & 800 & 0,429 & 700 & 0,181 & 600 & 0,136 \\
840 & 0,446 & 780 & 0,366 & 680 & 0,167 & 580 & 0,127 \\
830 & 0,463 & 760 & 0,304 & 660 & 0,160 & 560 & 0,111 \\
820 & 0,464 & 740 & 0,246 & 640 & 0,155 & & \\
\hline
\end{tabular}

Der Verlauf der Absorption zeigt, dass bei 820-830 nm mit der grössten Empfindlichkeit gemessen wird; die Empfindlichkeit genügt aber in den meisten Fällen auch noch dann, wenn ein Filterphotometer zur Verfügung steht, mit dem in der Gegend von $700 \mathrm{~nm}$ gemessen werden kann.

\subsection{Reproduzierbarkeit der Extinktionen in Standardreihen}

Die einzelnen Standardreihen sind im Laufe eines einzigen Tages angesetzt worden. Die verwendeten Reagenzlösungen sind am Morgen dieses Tages frisch zubereitet worden. Zeiss-Spektralphotometer PMQ II, $820 \mathrm{~nm}$, Schichtdicke $20 \mathrm{~mm}$.

\begin{tabular}{lllllllll}
\hline $\mathrm{mg} / 1 \mathrm{P}$ & $e$ & $e$ & $e$ & $e$ & $e$ & $e$ & $e$ & Mittelwert \\
\hline 0,025 & 0,044 & 0,042 & 0,038 & 0,040 & 0,038 & 0,037 & 0,037 & 0,039 \\
0,050 & 0,085 & 0,074 & 0,076 & 0,067 & 0,073 & 0,074 & 0,076 & 0,075 \\
0,10 & 0,147 & 0,149 & 0,151 & 0,146 & 0,147 & 0,149 & 0,147 & 0,148 \\
0,20 & 0,315 & 0,299 & 0,294 & 0,294 & 0,297 & 0,297 & 0,290 & 0,298 \\
\hline
\end{tabular}

Die Werte zeigten während Monaten nur um weniges grössere Streuungen. 


\subsection{Abhängigkeit der Extinktion von der Schwefelsäurekonzentration}

$0,2 \mathrm{mg} / 1 \mathrm{P}$. Durch verschieden grosse Schwefelsäurezugaben wurde die Konzentration der Messlösung an Schwefelsäure variiert. Die Angabe der Normalität an Schwefelsäure in der Tabelle bezieht sich auf die gesamte Mischung (= Messlösung, also $12 \mathrm{ml}$ ). Zeiss-Spektralphotometer PMQ II, $820 \mathrm{~nm}$, Schichtdicke $20 \mathrm{~mm}$.

\begin{tabular}{llll}
\hline Normalität & $e$ & Normalität & $e$ \\
\hline $1,00 n$ & 0,279 & $1,60 n$ & 0,286 \\
$1,20 n$ & 0,283 & $1,80 n$ & 0,276 \\
$1,40 n$ & 0,292 & $2,20 n$ & 0,024 \\
\hline
\end{tabular}

Nach unserer Arbeitsvorschrift ist das Gemisch an Schwefelsäure 1,2 $n$. In einem ziemlich weiten Konzentrationsbereich ist die Extinktion sehr wenig von der Schwefelsäurekonzentration abhängig. Das bestätigt die Angaben von BARTLETT [2] und die Zweckmässigkeit seines Vorgehens, auf die Neutralisation der vom Aufschluss her vorhandenen Schwefelsäure und das nachfolgend erneute Einmessen von Schwefelsäure (wie dies bei vielen anderen Arbeitsvorschriften zur Phosphatbestimmung verlangt wird) zu verzichten.

\subsection{Störung durch Eisen}

Das Eisen wurde als Eisen-III-ammoniumsulfat zugegeben. Beim Ansetzen der Testlösungen wurde zuerst die Schwefelsäure und dann der Eisenstandard zugegeben, um das Ausfallen von Eisenverbindungen zu vermeiden. Auf eine Störung durch Eisen-II wurde nicht geprüft, da Eisen in Abwässern und Vorflutern meist als EisenIII vorliegt und bei der Mineralisierung Eisen-II auf jeden Fall zu Eisen-III oxydiert wird. Zeiss-Spektralphotometer PMQ II, $820 \mathrm{~nm}$, Schichticke $10 \mathrm{~mm}$.

\begin{tabular}{llllll}
\hline $\mathrm{mg} / 1 \mathrm{P}$ & $\mathrm{mg} / 1 \mathrm{Fe}$ & $e$ & $\mathrm{mg} / 1 \mathrm{P}$ & $\mathrm{mg} / \mathrm{Fe}$ & $e$ \\
\hline 0,2 & 0 & 0,129 & 0,5 & 0 & 0,336 \\
0,2 & 0,5 & 0,135 & 0,5 & 0,5 & 0,335 \\
0,2 & 1,0 & 0,132 & 0,5 & 1,0 & 0,330 \\
0,2 & 2,0 & 0,121 & 0,5 & 2,0 & 0,321 \\
0,2 & 5,0 & 0,131 & 0,5 & 5,0 & 0,330 \\
0,2 & 10,0 & 0,125 & 0,5 & 10,0 & 0,325 \\
\hline
\end{tabular}

Die Bestimmung wird also durch Eisen-III in Konzentrationen bis zu 10 Milli gramm je Liter Messlösung nicht oder nur ganz unerheblich gestört.

\subsection{Störung durch Silikate}

Zubereitung der Silikatlösung: $1 \mathrm{~g}$ Kieselsäure gefällt, trocken «Merck» wurde im Platintiegel geglüht. Vom Glührückstand wurden $0,500 \mathrm{~g}$ mit $1,5 \mathrm{~g}$ wasserfreiem Natriumcarbonat im Platintiegel geschmolzen. Die Schmelze wurde in entsalztem Wasser zur $1000 \mathrm{ml}$ gelöst. Zeiss-Spektralphotometer PMQ II, $820 \mathrm{~nm}$, Schichtdicke $20 \mathrm{~mm}$. 


\begin{tabular}{llllll}
\hline $\mathrm{mg} / \mathrm{l} \mathrm{P}$ & $\mathrm{mg} / 1 \mathrm{SiO}_{2}$ & $e$ & $\mathrm{mg} / 1 \mathrm{P}$ & $\mathrm{mg} / 1 \mathrm{SiO}_{2}$ & $e$ \\
\hline 0 & 0 & - & 0,2 & 0 & 0,304 \\
0 & 2,0 & 0,007 & 0,2 & 2,0 & 0,298 \\
0 & 4,0 & 0,022 & 0,2 & 4,0 & 0,310 \\
\hline
\end{tabular}

Die Störung durch Silikate im untersuchten Konzentrationsbereich ist gering. Es ist im übrigen anzunehmen, dass die Silikate beim Mineralisieren zum grossen Teil als unlösliches Siliciumdioxid ausgeschieden werden.

\subsection{Weitere Störungen}

Arseniat gibt eine analoge Reaktion und stört daher erheblich. Eine grosse Anzahl störender Agenzien, vor allem organische Stoffe, werden durch die nasse Mineralisierung entfernt.

\subsection{Haltbarkeit des ANS-Reagenz}

Die im Reagenz gelöste 1-Amino-2-naphthol-4-sulfonsäure neigt dazu, beim Aufbewahren im Kühlschrank teilweise auszukristallisieren. Diese Erscheinung hat indessen innerhalb einer Woche nach unseren Beobachtungen keinen nennenswerten Einfluss auf die Reproduzierbarkeit der Extinktionswerte.

\subsection{Erfassbarkeit einiger organischer Phosphorverbindungen nach der Mineralisierung}

Als Testsubstanzen wurden Phosphorsäureester ausgewählt, da anzunehmen ist, dass die meisten im häuslichen Abwasser (und auch in vielen industriellen Abwässern, z.B. der Nahrungsmittelindustrie) vorkommenden organischen Phosphorverbindungen Phosphorsäureester sind.

\section{Testsubstanzen:}

I. Calciumglycerophosphat Ph. H.V.

II. Natriumglycerophosphat: $\beta$-Glycerinphosphatdinatriumsalz 95-96\% "Fluka». III. Adenosinmonophosphorsäure: Adenosin-5'-monophosphorsäuremonohydrat puriss. 99,5\% "Fluka».

IV. Glucosephosphat: D-Glucose-1-phosphat-Dikaliumsalz krist. puriss. $98 \%$ "Fluka».

Die Standardlösungen wurden so angesetzt, dass der P-Gehalt nach dem Mineralisieren theoretisch $5 \mathrm{mg} / \mathrm{P}$ betragen musste; die Aufschlusslösungen wurden zur Messung noch entsprechend verdünnt.

\begin{tabular}{cllrr}
\hline Substanz & mg/l P & (gefunden) & \multicolumn{2}{c}{$\%$ des theoretischen Wertes } \\
\hline I & 5,03 & 5,16 & $100,6 \%$ & $103,2 \%$ \\
II & 4,88 & 5,04 & $97,6 \%$ & $100,8 \%$ \\
III & 4,67 & 4,84 & $93,4 \%$ & $96,8 \%$ \\
IV & 4,64 & 4,73 & $92,8 \%$ & $94,6 \%$ \\
\hline
\end{tabular}

\subsection{Hinweis auf die Ausführung der nassen Mineralisierung}

$\mathrm{Da}$ in verschiedenen Laboratorien unterschiedliche Einrichtungen zum Erhitzen bei der Mineralisierung zur Verfügung stehen, empfiehlt es sich, dass der Analytiker sich bei der Einarbeitung in die Methode mit Einzelheiten in der Ausführung der 
nassen Mineralisierung vertraut macht. Der Einfluss von Temperatur und Dauer des Mineralisierens kann mit mehreren Ansätzen derselben Probe dadurch bestimmt werden, dass diese Bedingungen von Ansatz zu Ansatz variiert werden. Praktische Angaben zur Temperaturmessung machen Schmid und AмвüHL [5]. Auf die Temperaturmessung zwecks Normung der Einstellung der Wärmeapparate kann allerdings auch - unter Einhaltung der in der Arbeitsvorschrift angegebenen Arbeitsbedingungen - verzichtet werden. Die höchsten gefundenen Phosphatgehalte zeigen die besten Arbeitsbedingungen an.

\section{Zusammenfassung}

Es wird eine Arbeitsvorschrift zur Bestimmung des Gesamtphosphors in häuslichen bzw. kommunalen Abwässern, Kläranlagenabflüssen, auch nach P-Elimination, und in mit Abwasser verunreinigten Vorflutern angegeben. Bei industriellen Abwässern ist die Vorschrift, je nach der Art der darin enthaltenen Phosphorverbindungen, nicht in jedem Falle anwendbar. Störeinflüsse durch Eisen und Silikate wurden überprüft.

\section{SUMMARY}

A method is described for the determination of total phosphorus in domestic and municipal sewage as well as in effluents of sewage treatment plants, even after treatment for the elimination of phosphorus, and in polluted waters. The method is not in any case appropriate for industrial wastes. This depends on the nature of their phosphorus compounds. Interferences by iron and silicate have been studied.

\section{LITERATURVERZEICHNIS}

[1] АмвӥнL, H., und Schmid, M., Die Bestimmung geringster Mengen von Phosphation im Wasser von Binnenseen, Schweiz. Z. Hydrol. 27, 172 (1965).

[2] Bartlett, G. R., Phosphorus Assay in Column Chromatography, J. biol. Chem. 234, 466 (1959).

[3] Fiske, C. H., und Subbarow, Y., The Colorimetric Determination of Phosphorus, J. biol. Chem. 66, 375 (1925). J. biol. Chem. 66, 375 (1925).
[4] KLotTer, H. E., und NeusSEL, R., Orthophosphate und Polyphosphate im Abwasser
und Oberflächenwasser, Jb. Vom Wasser 26, 110 (1959).

[5] Sснміd, M., und АмвÜнL, H., Die Bestimmung geringster Mengen von Gesamtphosphor im Wasser von Binnenseen, Schweiz. Z. Hydrol. 27, 184 (1965).

[6] AASGP-Committee Report, Determination of Orthophosphate, Hydrolyzable Phosphate, and Total Phosphate in Surface Waters, J. Am. Water Wks Assoc. 50, 1563 (1958).

7] Standard Methods for the Examination of Water and Wastenwater, 11. Aufl. (Am. Public Health Assoc., New York 1960), S. 204.

Separatdruck aus der Schweiz. Zeitschrift für Hydrologie 29, Fasc. 2 (1967) Herausgegeben mit Unterstützung der Stiftung der Wirtschaft zur Förderung des Gewässerschutzes in der Schweiz 